\title{
TEMPERATURE EQUILIBRATION BEHIND THE SHOCK FRONT: AN OPTICAL AND X-RAY STUDY OF RCW 86
}

\author{
E. A. HELDER ${ }^{1}$, J. VINK ${ }^{1}$, AND C. G. BASSA ${ }^{2}$ \\ ${ }^{1}$ Astronomical Institute, Utrecht University, P.O. Box 80000, NL-3508 TA Utrecht, The Netherlands; e.a.helder@ astro-uu.nl \\ 2 Jodrell Bank Centre for Astrophysics, School of Physics and Astronomy, The University of Manchester, Oxford Road, Manchester M13 9PL, UK \\ Received 2011 February 2; accepted 2011 May 28; published 2011 August 8
}

\begin{abstract}
We study the electron-proton temperature equilibration behind several shocks of the RCW 86 supernova remnant. To measure the proton temperature, we use published and new optical spectra, all from different locations on the remnant. For each location, we determine the electron temperature from X-ray spectra, and correct for temperature equilibration between the shock front and the location of the X-ray spectrum. We confirm the result of previous studies that the electron and proton temperatures behind shock fronts are consistent with equilibration for slow shocks and deviate for faster shocks. However, we cannot confirm the previously reported trend of $T_{\mathrm{e}} / T_{\mathrm{p}} \propto 1 / v_{\mathrm{s}}^{2}$.
\end{abstract}

Key words: ISM: individual objects (RCW 86/MSH 14-63) - ISM: supernova remnants - radiation mechanisms: thermal - shock waves

\section{INTRODUCTION}

Shocks are ubiquitous in the universe and occur at various scales: from planetary shocks in our solar system to large-scale shocks in clusters of galaxies. Astrophysical shocks are rather different from shocks on Earth, as typical mean free paths for particle-particle interactions in the interstellar medium are of the order of parsecs, which is larger than the scale of the shock transitions of interstellar shocks. The fact that we observe sharp transitions implies that the existence of the shock must be communicated on scales shorter than this mean free path.

From conservation of mass, momentum, and energy over the shock front, and assuming an infinite Mach number and no cosmic-ray acceleration, the temperature $\left(T_{i}\right)$ of species with mass $m_{i}$ can be derived:

$$
k T_{i}=\frac{2(\gamma-1)}{(\gamma+1)^{2}} m_{i} v_{\mathrm{s}}^{2},
$$

in which $v_{\mathrm{s}}$ denotes the shock velocity, and $\gamma$ the equation of state of the plasma $(\gamma=5 / 3$ for a non-relativistic gas). This equation holds for a plasma maximally out of thermal equilibrium, implying an electron temperature over proton temperature $\left(T_{\mathrm{e}} / T_{\mathrm{p}}\right)$ of the ratio of the electron and proton masses $\left(m_{\mathrm{e}} / m_{\mathrm{p}}=1 / 1836\right)$. For a plasma in thermal equilibrium, Equation (1) reads

$$
k T=\left\langle k T_{i}\right\rangle=\frac{2(\gamma-1)}{(\gamma+1)^{2}} \mu m_{\mathrm{p}} v_{\mathrm{s}}^{2}
$$

in which $\mu$ is the mean particle mass ( 0.6 for a fully ionized plasma with solar abundances). However, if a shock is accelerating cosmic rays, a cosmic-ray precursor will develop, which heats the incoming medium and lowers the effective Mach number of the shock, in which case Equations (1) or (2) are no longer valid (see also Drury et al. 2009; Vink et al. 2010).

In most empirical studies on temperature equilibration behind supernova remnant shocks, $T_{\mathrm{e}}$ and $T_{\mathrm{p}}$ are determined from Balmer-dominated shocks. These shocks are characterized by hydrogen line emission in the Balmer series, leading to a line profile consisting of two superimposed lines. These shocks are non-radiative; their energy losses in the form of radiation are dynamically negligible (this happens for shock velocities
$>200 \mathrm{~km} \mathrm{~s}^{-1}$; e.g., Draine \& McKee 1993). This implies that the hydrogen lines of these shocks are caused by impact excitation, not by recombination. The narrow component of the spectrum is emitted by neutral hydrogen atoms, excited shortly after entering the shock front; its width reflects the temperature of the ambient medium. The broad component is emitted after charge exchange between incoming neutral hydrogen and hot protons behind the shock front. The width reflects the temperature of the protons behind the shock front. Combining the width with the ratio of the flux in the narrow to the flux in the broad component, one can determine the shock velocity and hence the electron temperature (for a recent review, see Heng 2010). This procedure works for shocks which have negligible cosmic-ray acceleration. If a shock is an efficient accelerator, the cosmic-ray precursor ionizes and heats the incoming medium and therewith increases the flux of the narrow component (Raymond et al. 2011).

The electron temperature for most young supernova remnants can also be measured from the X-ray spectrum, as the thermal component of the X-ray spectrum is shaped by the electron temperature. Electron temperatures measured from X-ray spectra are generally not the electron temperatures right behind the shock front, but rather somewhat further to the inside of the shock, where temperatures have already (partially) equilibrated through Coulomb interactions. The amount of equilibration is a function of $n_{\mathrm{e}} t$, the ionization age, that can be determined from the X-ray spectrum simultaneously with the electron temperature, from the ratio of the fluxes in different spectral lines from the same species.

Rakowski et al. (2003) studied shocks of the supernova remnant DEM L71 at both optical and X-ray wavelengths. The remnant has shock velocities between 555 and $980 \mathrm{~km} \mathrm{~s}^{-1}$. Correcting for temperature equilibration effects, Rakowski et al. (2003) showed that $T_{\mathrm{e}} / T_{\mathrm{p}}$ decreases with increasing shock velocity.

From a theoretical point of view, it is not well determined how much electron-proton temperature equilibration would be expected behind a shock front. Empirical studies of shocks with negligible cosmic-ray acceleration show that electron and proton temperatures are equilibrated for slow shocks $\left(v_{\mathrm{s}}<\right.$ $\left.400 \mathrm{~km} \mathrm{~s}^{-1}\right)$, whereas the degree of thermal equilibration decreases for faster shocks (Rakowski et al. 2003; Ghavamian et al. 2007; van Adelsberg et al. 2008). More specifically, a 
relation of $T_{\mathrm{e}} / T_{\mathrm{p}} \propto v_{\mathrm{s}}^{-2}$ has been suggested for shocks with $v_{s}>$ $400 \mathrm{~km} \mathrm{~s}^{-1}$ (Ghavamian et al. 2007). This directly implies that $T_{\mathrm{e}}$ is nearly constant closely behind the shock front (i.e., apart from temperature equilibration effects, Equation (2) in Ghavamian et al. 2002), independent of the shock velocity (Equation (1)). The origin of this relation is attributed by Ghavamian et al. (2007) to so-called lower hybrid waves ahead of the shock, caused by a (moderate) cosmic-ray precursor. Note that van Adelsberg et al. (2008) could not reproduce $T_{\mathrm{e}} / T_{\mathrm{p}} \propto v_{\mathrm{s}}^{-2}$, also using published data of $\mathrm{H} \alpha$ spectra, but with different models for interpreting $\mathrm{H} \alpha$ spectra. Bykov \& Uvarov (1999) and Bykov (2004) point out that for Mach numbers $M<\sqrt{m_{\mathrm{p}} / m_{\mathrm{e}}}=43$ the electrons ahead of the shock have a typical thermal velocity exceeding the shock velocity, which may lead, through nonresonant interactions with large-amplitude turbulent fluctuations in the shock transition region, to collisionless electron heating and acceleration. Interestingly, assuming a typical sound speed of the ambient medium of $10 \mathrm{~km} \mathrm{~s}^{-1}, M=43$ corresponds to a shock speed of $\sim 400 \mathrm{~km} \mathrm{~s}^{-1}$. For a cosmic-ray accelerating shock it is even more complex, as the incoming material is already pre-heated by a cosmic-ray precursor, leading to a lower Mach number at the main shock. This could mean that for cosmic-ray-dominated shocks the electron-proton temperatures could be equilibrated again.

Here, we investigate the electron-proton temperature equilibration behind several shock fronts of RCW 86, using $\mathrm{X}$-ray (XMM-Newton/EPIC (European Photon Imaging Camera) MOS (Metal-Oxide-Silicon)) and optical (Very Large Telescope (VLT)/FORS2) data. The shocks of the supernova remnant RCW 86 are particularly well suited for this study, as the ionization age of the plasma in parts of the remnant is low: $\sim 5 \times 10^{9} \mathrm{~cm}^{-3}$ s (e.g., Vink et al. 1997); this value is low compared to the ionization age of most other remnants. ${ }^{3}$ The low value for $n_{\mathrm{e}} t$ makes the measured electron temperature a more direct indication of the electron temperature at the shock front.

Additionally, the shocks of RCW 86 have a large variety in speeds, as the progenitor exploded in its own wind-blown bubble (e.g., Vink et al. 1997) and parts of the remnant have already hit the dense rim of this bubble. As a result, the southwest (SW) rim has slowed down; its X-ray emission is mostly thermal and the shock velocity is $\sim 500 \mathrm{~km} \mathrm{~s}^{-1}$ (Ghavamian et al. 2001). The $\mathrm{X}$-ray emission of the northeast (NE) shock is dominated by $\mathrm{X}$-ray synchrotron emission (Vink et al. 2006), and its shock velocity is measured to be $6000 \pm 2800 \mathrm{~km} \mathrm{~s}^{-1}$ (Helder et al. 2009). However, its proton temperature is low, which is an indication for efficient cosmic-ray acceleration.

Furthermore, the mostly sub-solar abundances of the thermal $\mathrm{X}$-ray emission at the shock front of RCW 86 indicate emission from shocked ambient medium (rather than shocked ejecta, e.g., Vink et al. 1997; Borkowski et al. 2001; Rho et al. 2002), tying the fitted parameters of the X-ray spectrum to the physics at the shock front.

\section{DATA AND RESULTS}

\subsection{VLT/FORS2}

In this study, we use both published and new optical observations. The observation taken from the SW of the remnant was previously published in Ghavamian et al. (2001) and the observation from the NE rim was published in Helder et al. (2009).

\footnotetext{
3 A plasma can be regarded as being in collisional equilibration for $n_{\mathrm{e}} t$ of $\gtrsim 10^{12} \mathrm{~cm}^{-3} \mathrm{~s}$.
}

Table 1

Widths of Broad Components of $\mathrm{H} \alpha$ Lines at the Different Locations and Corresponding Post-shock Proton and Electron Temperatures

\begin{tabular}{lccc}
\hline \hline Location & $\begin{array}{c}\text { Broad } \mathrm{H} \alpha \text { Width } \\
\left(\mathrm{km} \mathrm{s}^{-1}\right)\end{array}$ & $\begin{array}{c}\text { Proton } T \\
(\mathrm{keV})\end{array}$ & $\begin{array}{c}\text { Electron } T^{\mathrm{a}} \\
(\mathrm{keV})\end{array}$ \\
\hline $\mathrm{NE}$ & $1100 \pm 60^{\mathrm{b}}$ & $2.3 \pm 0.3^{\mathrm{a}}$ & $>1.5$ \\
$\mathrm{SE}_{\text {in }}$ & $920 \pm 50^{\mathrm{c}}$ & $1.6 \pm 0.2^{\mathrm{b}}$ & $0.75 \pm 0.15$ \\
$\mathrm{SE}_{\text {out }}$ & $1120 \pm 40^{\mathrm{c}}$ & $2.4 \pm 0.2^{\mathrm{b}}$ & $<0.05$ \\
$\mathrm{SW}$ & $562 \pm 18^{\mathrm{d}}$ & $0.60 \pm 0.04^{\mathrm{c}}$ & $<0.05$ \\
$\mathrm{~N}$ & $325 \pm 10^{\mathrm{e}}$ & $0.2 \pm 0.02$ & $1.95 \pm 0.23$ \\
$\mathrm{~N}$ & $680 \pm 70^{\mathrm{f}}$ & $0.9 \pm 0.2^{\mathrm{c}}$ & $1.0 \pm 0.2$ \\
$\mathrm{E}$ & $640 \pm 35^{\mathrm{e}}$ & $0.77 \pm 0.04$ & $0.78 \pm 0.13$ \\
$\mathrm{NW}$ & $580 \pm 18^{\mathrm{g}}$ & $0.64 \pm 0.04$ & $0.27 \pm 0.08$ \\
\hline
\end{tabular}

Notes.

a At the shock front, derived in this study.

${ }^{b}$ Helder et al. (2009).

c Derived in this study.

d Ghavamian et al. (2001).

e Ghavamian et al. (2007).

${ }^{\mathrm{f}}$ Long \& Blair (1990).

g Ghavamian (1999).

The parameters of the north $(\mathrm{N})$ and east $(\mathrm{E})$ observations are taken from Ghavamian et al. (2007) and the northwest (NW) location from Ghavamian (1999). The exact locations of the $\mathrm{N}$ and E spectra have been kindly provided to us by P. Ghavamian (2010, private communication). We also use parameters of a spectrum published in Long \& Blair (1990); this spectrum is taken from the northern region as well. Table 2 summarizes the line widths used in the present paper.

The optical image of Figure 1 has been obtained with VLT/ FORS2 as pre-image for the spectral observation, both in 2007 (programme ID 079.D-0735). The image is a combination of three exposures of $200 \mathrm{~s}$, through a narrowband $\mathrm{H} \alpha$ filter (H_Alpha+83). To correct for stellar light, three similar images through a narrowband $\mathrm{H} \alpha$ filter, shifted $4500 \mathrm{~km} \mathrm{~s}^{-1}$ (H_Alpha/4500+61), have been taken.

Figure 1 shows the location of the slit used to obtain the spectra. The spectra of the southeast (SE) part of the remnant were taken in a single 2733 s exposure, using a 1200R grism and a 2 .'5 slit. This setup resulted in a spectral resolution of $350 \mathrm{~km} \mathrm{~s}^{-1}$, which is sufficient for resolving the broad component; however, the narrow component of the line probably remains unresolved. The data were reduced with standard data reduction steps, as described in Helder et al. (2009), resulting in the spectra shown in Figure 2.

The slit crossed several filaments (Figure 1), and we here present spectra of the outer and inner filaments. We focus on the line width of the broad component, as this is directly related to the post-shock proton temperature. We fit the spectra with two Gaussian lines, convolved with the resolution of the instrument setup. Table 1 lists the line widths obtained from the eastern $\left(\mathrm{SE}_{\text {out }}\right)$ and western $\left(\mathrm{SE}_{\mathrm{in}}\right)$ spectra in the slit (Figure 1). As the signal to noise of the spectrum of the innermost filament is rather low, we used both the inner and middle filaments for the $\mathrm{SE}_{\mathrm{in}}$ spectrum. A fit to the innermost filament alone revealed a width of the broad line similar to the width found for the combined spectrum.

\subsection{XMM-Newton}

We determined electron temperatures from X-ray spectra taken with the XMM-Newton/EPIC MOS instruments (Turner et al. 2001). We chose the MOS instruments as they have 


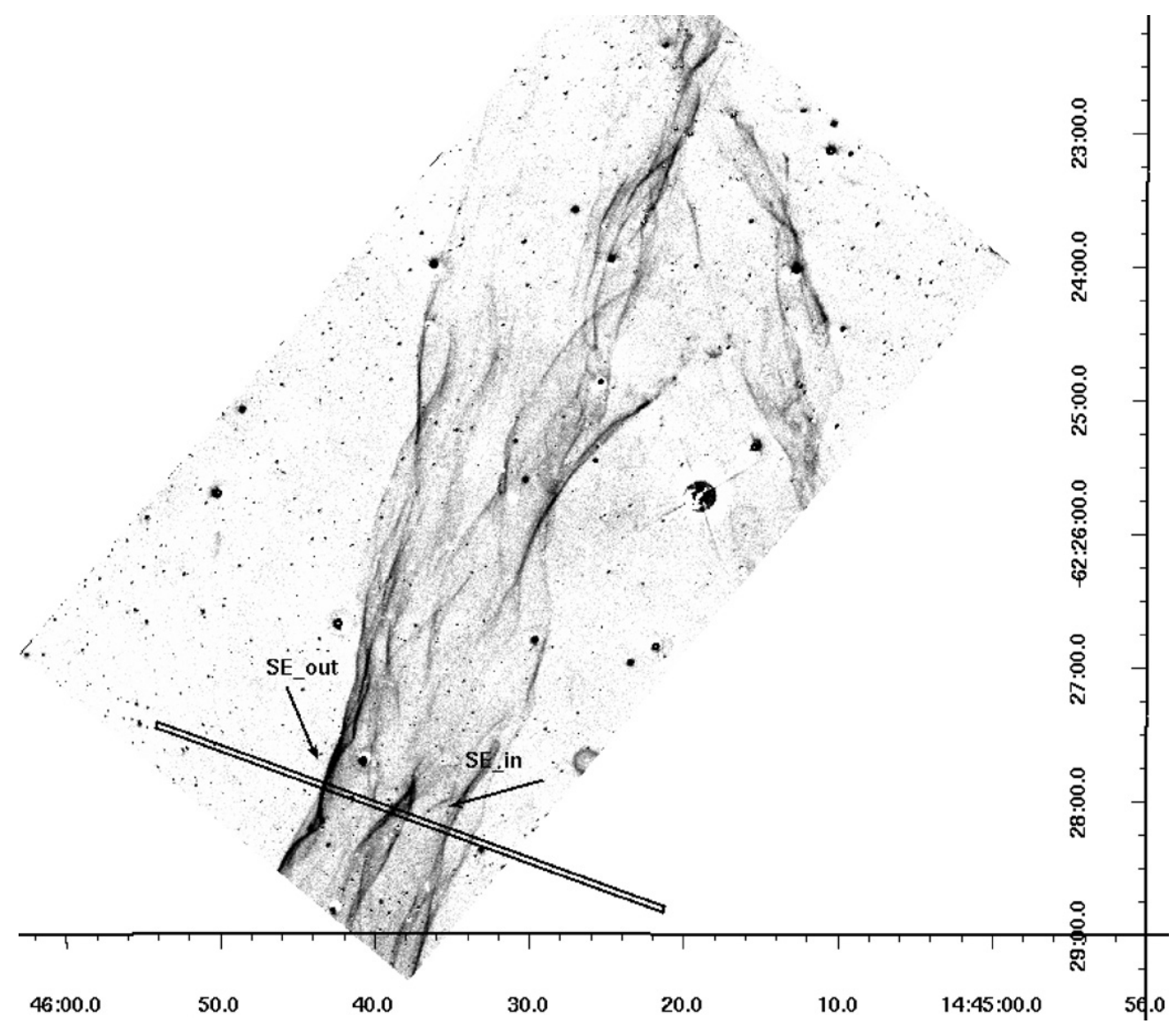

Figure 1. Southwest $\mathrm{H} \alpha$ filament of RCW 86, as observed with VLT/FORS2. Overlaid is the slit position used for the spectra in Figure 2.
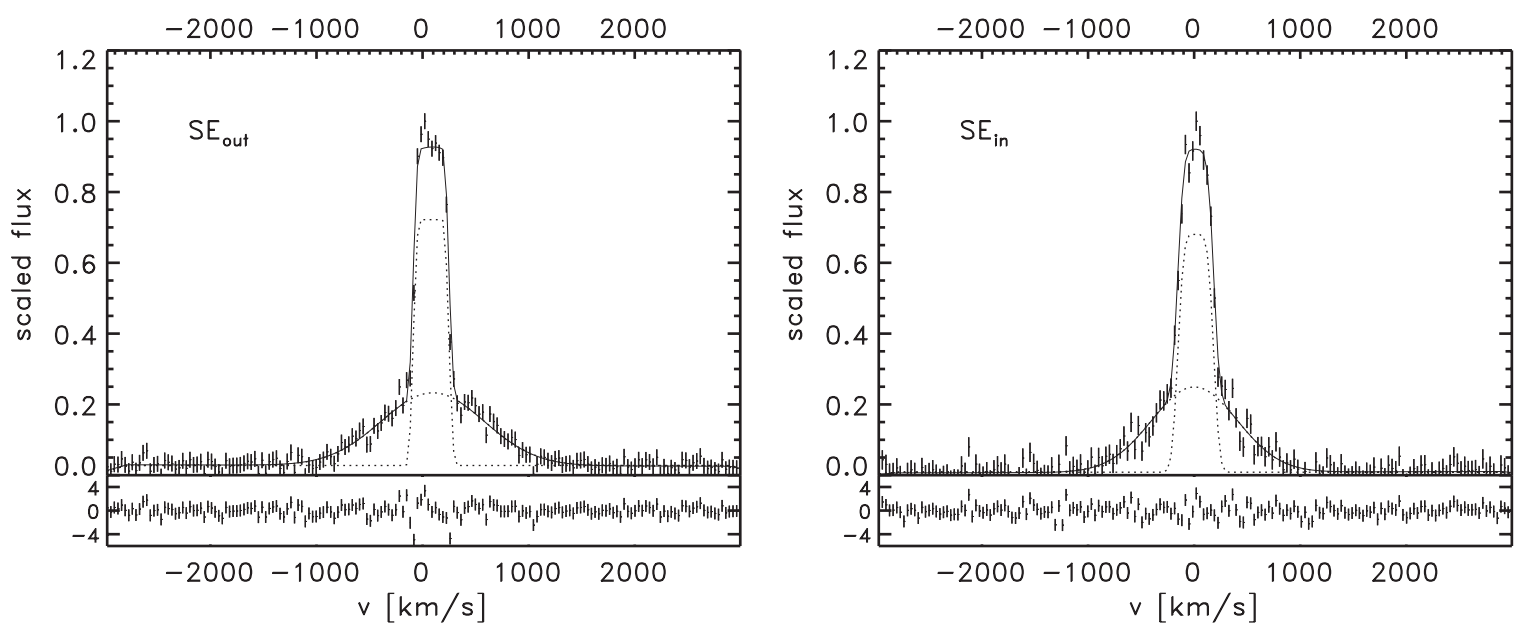

Figure 2. $\mathrm{H} \alpha$ line in the spectrum of the $\mathrm{SE}_{\text {out }}$ region (left) and for the $\mathrm{SE}_{\text {in }}$ region (right). Overplotted in both figures are the individual broad and narrow components (dotted lines) and the total fit (solid line).

a higher spectral resolution than the XMM-Newton/EPIC pn CCDs (Strüder et al. 2001). The observations for the $\mathrm{E}, \mathrm{SE}_{\text {in }}$, and $\mathrm{SE}_{\text {out }}$ spectra were taken on August 13 (ObsID 0504810201, $75 \mathrm{ks}$ ), the NE on July 28 (ObsID 0504810101, $117 \mathrm{ks}$ ), the $\mathrm{N}$ and NW on 2007 August 25 (ObsID 0504810301, 74 ks), and for the SW spectrum on 2007 August 23 (ObsID 0504810401, $73 \mathrm{ks}$ ). The spectra (shown in Figure 4) were extracted at locations close to the regions for which we have $\mathrm{H} \alpha$ spectra, as indicated in Figure 3, using the XMM SAS data reduction software, version 1.52.8. Unfortunately, the position angle of the XMM telescope was chosen such that only the EPIC MOS2 instrument observed the region of interest for the NE, NW, and $\mathrm{SW}$ rims. For the other spectra, we used both the MOS1 and MOS2 instruments.

We fitted the extracted spectra with a non-equilibrium ionization (NEI) model (Kaastra \& Jansen 1993), combined with an absorption model, using the SPEX spectral fitting software version 10.0.0 (Kaastra et al. 1996), and using the maximum likelihood statistic for Poisson distributions ( $C$-statistic; Cash 1979). This statistic is more appropriate than the classical $\chi^{2}$-method for fitting spectra which contain bins with few counts (Wheaton et al. 1995). For more counts, this statistic 
Table 2

The Best-fit Parameters for XMM-Newton MOS Spectra

\begin{tabular}{|c|c|c|c|c|c|c|c|}
\hline Parameter & $\mathrm{NE}^{\mathrm{a}}$ & $\mathrm{SE}_{\text {in }}{ }^{\mathrm{b}}$ & $\mathrm{SE}_{\text {out }}{ }^{\mathrm{b}}$ & $\mathrm{SW}^{\mathrm{a}, \mathrm{c}}$ & $\mathrm{E}^{\mathrm{b}}$ & $\mathrm{N}^{\mathrm{b}}$ & $\mathrm{NW}^{\mathrm{a}}$ \\
\hline$\overline{\mathrm{EM}^{\mathrm{d}}}$ & $0.13 \pm 0.05$ & $4.4 \pm 0.7$ & $4 \pm 2$ & $360 \pm 240$ & $6.0 \pm 0.7$ & $4.2 \pm 0.3$ & $20_{-9}^{+80}$ \\
\hline$k T_{\mathrm{e}}(\mathrm{keV})$ & $37_{-19}^{+27}$ & $0.9 \pm 0.1$ & $0.6 \pm 0.1$ & $0.18 \pm 0.01$ & $0.8 \pm 0.1$ & $1.0 \pm 0.2$ & $0.27 \pm 0.08$ \\
\hline$n_{\mathrm{e}} t\left(10^{9} \mathrm{~cm}^{-3} \mathrm{~s}\right)$ & $0.24 \pm 0.03$ & $7.6 \pm 0.7$ & $6 \pm 1$ & $6.9 \pm 0.5$ & $4.2 \pm 0.7$ & $3.1 \pm 0.5$ & $2.0_{-0.7}^{+4}$ \\
\hline $\mathrm{O}^{\mathrm{e}}$ & $1^{\mathrm{f}}$ & $0.47 \pm 0.03$ & $0.46 \pm 0.05$ & $0.51 \pm 0.2$ & $0.52 \pm 0.2$ & $0.38 \pm 0.05$ & 1 \\
\hline $\mathrm{Ne}$ & 1 & $0.63 \pm 0.05$ & $0.52 \pm 0.07$ & $4.14 \pm 1.6$ & $0.54 \pm 0.02$ & $0.44 \pm 0.06$ & 1 \\
\hline $\mathrm{Mg}$ & 1 & $0.40 \pm 0.05$ & $0.25 \pm 0.08$ & $0.7_{-0.7}^{+1.4}$ & $0.27_{-0.05}^{+0.2}$ & $0.15 \pm 0.09$ & 1 \\
\hline $\mathrm{Fe}$ & 1 & $0.5 \pm 0.1$ & 1 & $298_{-91}^{+153}$ & $2.7 \pm 0.8$ & $2.6_{-1.6}^{+5}$ & 1 \\
\hline PL norm. ${ }^{g}$ & $7.0 \pm 0.3$ & $\ldots$ & $\ldots$ & $\ldots$ & $\ldots$ & $\ldots$ & $\ldots$ \\
\hline$\Gamma$ & $2.9 \pm 0.1$ & $\ldots$ & $\ldots$ & $\ldots$ & $\ldots$ & $\ldots$ & $\ldots$ \\
\hline$N_{\mathrm{H}}\left(10^{21} \mathrm{~cm}^{-2}\right)$ & $4.7 \pm 0.2$ & $2.8 \pm 0.2$ & $3.6 \pm 0.4$ & $5.1 \pm 0.4$ & $3.6 \pm 0.2$ & $4.2 \pm 0.3$ & $5.5 \pm 1$ \\
\hline$C$-statistics/dof & $131 / 117$ & $347 / 136$ & $197 / 137$ & $145 / 88$ & $202 / 137$ & $256 / 136$ & $84 / 52$ \\
\hline
\end{tabular}

Notes. The errors are $1 \sigma$.

${ }^{\text {a }}$ MOS2 data.

b Based on both MOS1 and MOS2 data.

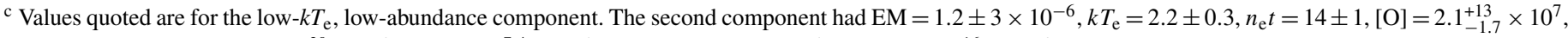
$[\mathrm{Ne}]=(6 \pm 3) \times 10^{7},[\mathrm{Mg}]=6_{-4}^{+20} \times 10^{6},[\mathrm{Si}]=3.4_{-3.4}^{+7.4} \times 10^{6},[\mathrm{~S}]=(2.5 \pm 1.8) \times 10^{6}$, and $[\mathrm{Fe}]=4_{-1.7}^{+16} \times 10^{6}$. All units are the same as in the table.

$\mathrm{d}$ The emission measure (EM) is defined as $\int n_{\mathrm{e}} n_{\mathrm{H}} d V / d^{2}$ in units $10^{55} \mathrm{~cm}^{-3}(2.5 \mathrm{kpc})^{-2}$.

e Abundances are relative to solar values (Anders \& Grevesse 1989).

f A " 1 " indicates abundances fixed to solar abundances.

g Power-law normalization $\left(10^{44} \mathrm{ph} \mathrm{s}^{-1} \mathrm{keV}^{-1}\right)$.

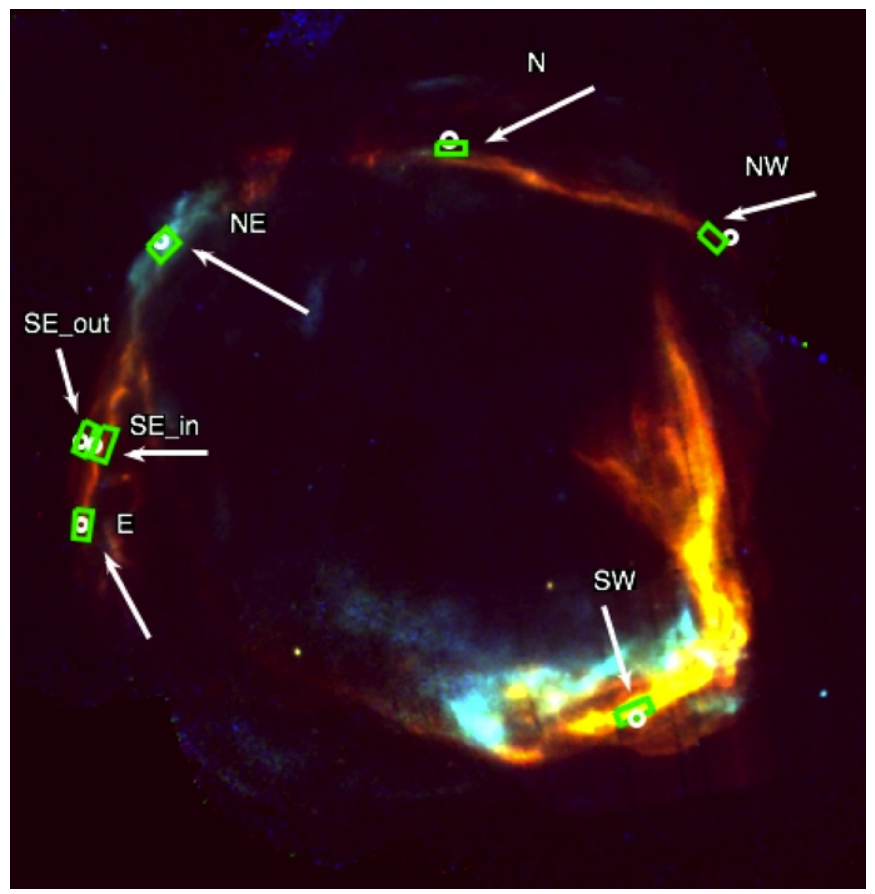

Figure 3. RGB image obtained with XMM-Newton. Shown are the regions from which the $\mathrm{H} \alpha$ lines have been obtained (white circles). The green boxes show regions from which we extracted the corresponding X-ray spectra. In this image, red indicates $0.5-1.0 \mathrm{keV}$, green $1.0-2.0 \mathrm{keV}$, and blue $2.0-4.0 \mathrm{keV}$.

asymptotically approaches the $\chi^{2}$-statistic. For most of the spectra, this method was relatively straightforward. Some regions needed some special attention, as described below. The NE spectrum needed an additional power-law component to account for the synchrotron emission present in the spectrum (Vink et al. 2006). Including the power law to account for the synchrotron emission results in an ill-constrained electron temperature, with

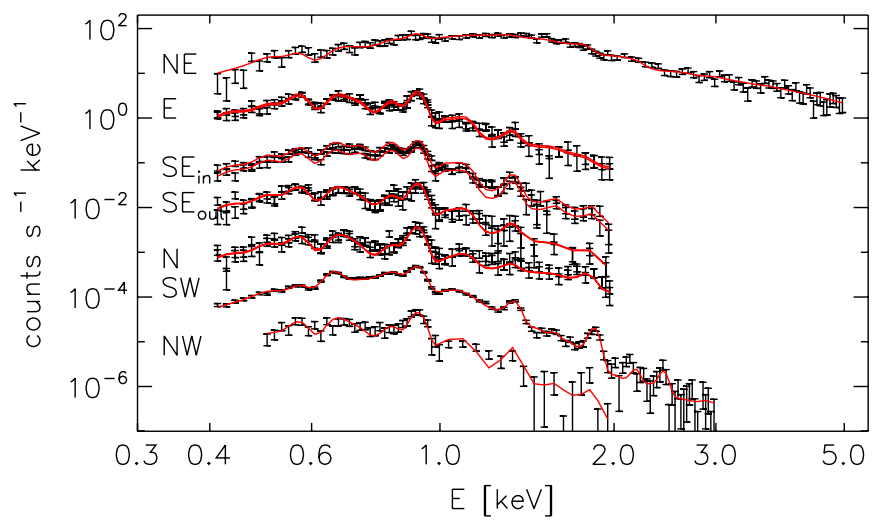

Figure 4. Spectra corresponding to the regions from Figure 3. Red lines are the best-fitting models. The NE spectrum is multiplied by $1000, \mathrm{E}$ by $20, \mathrm{SE}_{\text {in }}$ by $1, \mathrm{SE}_{\text {out }}$ by $0.3, \mathrm{~N}$ by $0.06, \mathrm{SW}$ by 0.0003 , and $\mathrm{NW}$ by 0.0007 .

a nominal electron temperature of $37 \mathrm{keV}$. However, the continuum emission is dominated by synchrotron radiation and the temperature is therefore mostly determined by the very weak line emission. Vink et al. (2006) encountered similar problems but concentrated on a slightly different extraction region, which had more thermal emission. They reported $T_{\mathrm{e}}=6.7 \pm 2.6 \mathrm{keV}$. The high, but ill-constrained electron temperature we found in this study and the high value reported by Vink et al. (2006) suggest that the electron and proton temperatures are likely close to equilibration. However, in order to be conservative, we take now the $2 \sigma$ lower limit of Vink et al. $2006(1.5 \mathrm{keV})$ as a lower limit on the electron temperature.

The SW spectrum cannot be fitted adequately with a single NEI component (Cash statistic/dof = 6.4). Subsequent analysis with two NEI components with approximately solar abundances, which we coupled for the components, resulted in a Cash statistic/dof $=4.5$. We carried out a different 


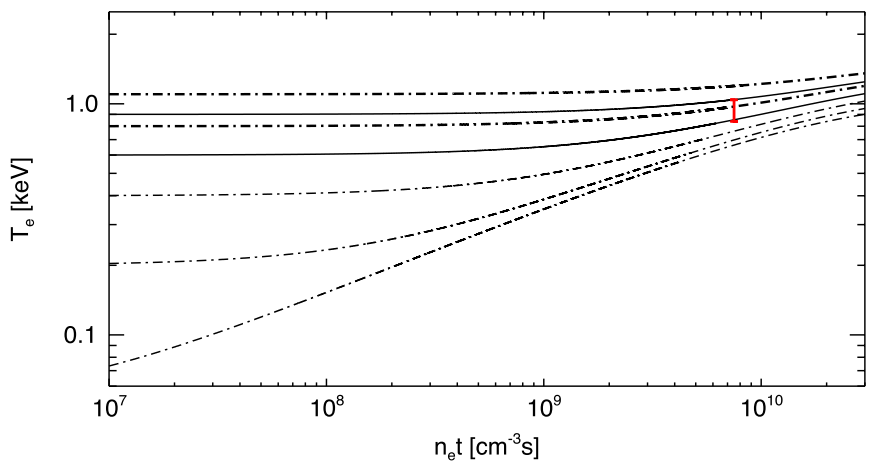

Figure 5. Temperature histories for the $\mathrm{SE}_{\text {in }}$ location $\left(T_{\mathrm{p}}=1.6 \mathrm{keV}\right)$ as functions of $n_{\mathrm{e}} t$ for different $T_{\mathrm{e}}$ values at the shock front. From top to bottom, the lines indicate a $T_{\mathrm{e}}$ at the shock front of $1.10,0.95,0.80,0.60,0.40,0.20$, and $0.05 \mathrm{keV}$.

approach by constructing a second NEI component, consisting of pure metals. We constructed this component by increasing the abundances of $\mathrm{O}, \mathrm{Ne}, \mathrm{Mg}, \mathrm{Si}, \mathrm{S}$, and Fe by a factor of $10^{7}$, mimicking a pure metal plasma (a similar procedure was adopted for the 0519-69.0 remnant in the LMC; Kosenko et al. 2010). We found Cash statistic/dof $=1.6$ for this approach. We found a high $T_{\mathrm{e}}(2.2 \pm 0.3 \mathrm{keV})$ and $n_{\mathrm{e}}\left(1.4 \pm 0.1 \times 10^{10} \mathrm{~cm}^{-3} \mathrm{~s}\right)$ for the pure metal plasma component, and a low $T_{\mathrm{e}}$ and $n_{\mathrm{e}}$ for the other component (Table 2). This, together with the complex, layered structure of the remnant in the SW, indicates that the pure metal component possibly represents a shocked ejecta component in the spectrum and the low-metallicity component is probably tied to the shocked ambient medium. We note that a strong ejecta component with alpha elements has not been reported before for this remnant, but several studies suggested the presence of Fe-rich ejecta giving rise to low ionization Fe-K lines (Bamba et al. 2000; Borkowski et al. 2001; Yamaguchi et al. 2008). Also note that this solution is not necessarily unique but, given the high $C$-statistic values for the alternative models, we regard this model as an adequate representation of the spectrum. A followup study with a deeper observation is necessary to shed further light on the emission from this region and to investigate whether there is indeed an ejecta component present in this region.

The $\mathrm{SE}_{\text {out }}$ spectrum is fitted with the Fe abundance tied to unity, as otherwise this abundance would rise to unrealistically high values. We note that releasing this component during the fitting procedure will result in an even lower electron temperature $(0.3 \mathrm{keV})$.

Table 2 lists the parameters of the best-fitting spectral model.

\section{DISCUSSION}

\subsection{XMM Spectra}

The parameters in Table 2 are in general consistent with parameters obtained in previous work (Rho et al. 2002; Vink et al. 2006). In the NE, the dominant contribution of synchrotron radiation makes it difficult to determine the electron temperature adequately. Our plasma parameters for the SW spectrum differ from those previously determined (Rho et al. 2002), and this is probably because we fit the spectrum with two components, of which one probably represents the ejecta and one the shocked ambient medium, whereas Rho et al. used a single component. We choose our region right behind a Balmerdominated filament, which is non-radiative and hence has a relatively low density. Additionally, the plasma from the ambient medium was probably only recently shocked. The low value for

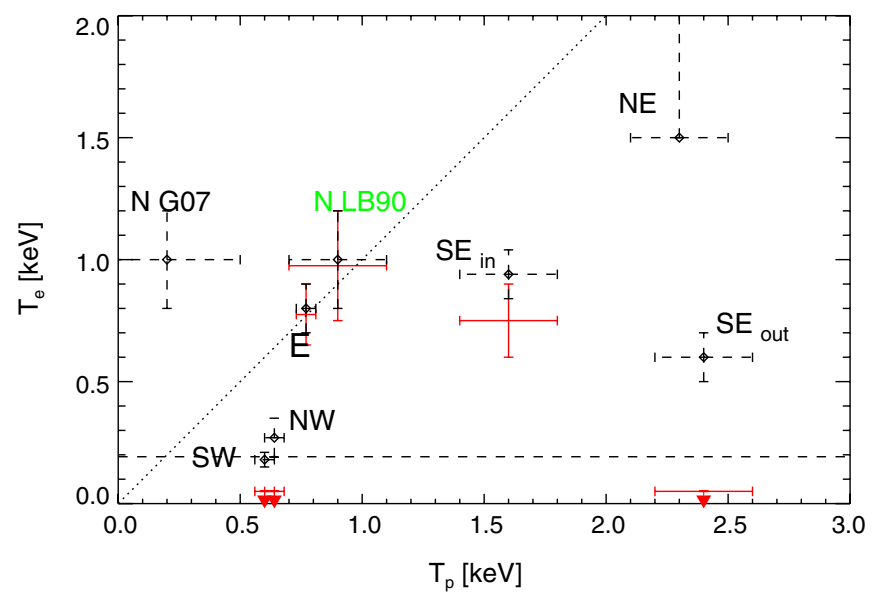

Figure 6. Proton and electron temperatures ( $x$ - and $y$-axes, respectively) from Tables 1 and 2. The black data points show the electron temperature as calculated from the X-ray spectra. The red data points indicate electron temperatures at the shock front after correcting for Coulomb equilibration. The dotted line indicates $T_{\mathrm{p}}=T_{\mathrm{e}}$. The dashed line indicates the electron temperature as calculated for a $400 \mathrm{~km} \mathrm{~s}^{-1}$ shock with thermal equilibrium behind the shock front; the relation found by Ghavamian et al. (2007) would be on this line. The points labeled "N G07" and "N LB90" refer to the same region in the north, with $T_{\mathrm{p}}$ based on the parameters of Ghavamian et al. (2007) and Long \& Blair (1990), respectively.

the $n_{\mathrm{e}} t$ for the ambient medium component therefore seems appropriate. Alternatively, if we choose our temperature similar to Rho et al. (2002), $T_{\mathrm{e}}$ and $T_{\mathrm{p}}$ are close to equilibration.

Problems with fitting the spectrum around $1.2 \mathrm{keV}$ were also encountered by Kosenko et al. (2008) for the 0509-67.5 supernova remnant. These deviations are possibly caused by uncertainties in the atomic database of SPEX, presumably by the Fe-L line complex.

The sub-solar abundances in all fits show that we indeed fit $\mathrm{X}$-ray spectra from a shocked ambient medium as opposed to metal-enhanced shocked ejecta. This confirms that the measured electron temperatures are related to the proton temperatures at the shock front.

\subsection{Proton Temperatures}

The width of the broad component is primarily a function of the post-shock proton temperature, but modified by the velocitydependent cross sections for charge and impact excitation. This causes the shape of the broad component to deviate from a perfect Gaussian. Nevertheless this remains a decent approximation (see van Adelsberg et al. 2008, and references therein). Figure 5 of van Adelsberg et al. (2008) shows that up to $2000 \mathrm{~km} \mathrm{~s}^{-1}$, the FWHM of the broad line increases linearly with the shock velocity. This shows that interpreting the line width in terms of proton temperature through $k T_{\mathrm{p}}=m_{\mathrm{p}} \sigma^{2}$ is a decent approximation (with $\sigma=\mathrm{FWHM} / \sqrt{8 \ln 2}$ in $\mathrm{km} \mathrm{s}^{-1}$; Rybicki \& Lightman 1979; Heng 2010; Table 1).

Table 1 shows that $T_{\mathrm{p}}$ for $\mathrm{SE}_{\text {out }}$ is higher than $T_{\mathrm{p}}$ for the $\mathrm{SE}_{\text {in }}$ spectrum, implying a higher shock velocity (Equation (1)). Note that $\mathrm{SE}_{\text {out }}$ lies outward of $\mathrm{SE}_{\mathrm{in}}$, which is suggestive of a higher shock velocity as well. For the northern region of RCW 86 , we have the parameters of two $\mathrm{H} \alpha$ spectra (Long \& Blair 1990; Ghavamian et al. 2007) at nearly the same location, which appear to have significantly different proton temperatures $(0.2$ and $0.9 \mathrm{keV}$, respectively). As we do not know which value best represents this region, we plotted both values for $T_{\mathrm{p}}$ with one corresponding $T_{\mathrm{e}}$ in Figure 6. We note that the measured widths of the broad $\mathrm{H} \alpha$ lines tend to vary a lot (from 325 to 
$543 \mathrm{~km} \mathrm{~s}^{-1}$ ) in the northern region (Ghavamian 1999), making it unclear to which $T_{\mathrm{p}}$ the electron temperature relates (probably a combination). Also, we note that $T_{\mathrm{p}}$ appears to vary significantly along the eastern rim of the remnant (Ghavamian 1999).

\subsection{Temperature Equilibration at the Shock Front}

The proton temperature is obtained from optical spectra directly behind the shock front, as the neutral hydrogen will ionize quickly after entering the shock front. To obtain an X-ray spectrum with sufficient signal over noise, we extracted over a larger region of the remnant than the region from which the proton temperature was determined. Also, the spatial resolution of the EPIC MOS instruments $\left(6^{\prime \prime}\right)$ prevents us from extracting an X-ray spectrum from very close to the shock front.

This means that the electron and proton temperatures will have undergone some equilibration at the extraction region of the X-ray spectrum, implying that the electron temperatures obtained are upper limits (black data points in Figure 6). To compare this to the proton temperatures behind the shock front, we need to determine the amount of heating the electrons experienced. We assume that the electrons are heated solely by Coulomb interactions. Spitzer (1965) derived the coupled differential equation for how fast species $i$ and $j$ with different temperatures $\left(T_{i}\right.$ and $\left.T_{j}\right)$ equilibrate as function of time $(t)$ :

$$
\frac{d T_{i}}{d t}=\frac{T_{j}-T_{i}}{t_{\mathrm{eq}(i, j)}}, t_{\mathrm{eq}(i, j)}=5.87 \frac{A_{i} A_{j}}{n_{j} Z_{i}^{2} Z_{j}^{2} \ln (\Lambda)}\left(\frac{T_{i}}{A_{i}}+\frac{T_{j}}{A_{j}}\right)^{\frac{3}{2}},
$$

for species with atomic numbers $A_{i}$ and $A_{j}$, charge $Z_{i}$ and $Z_{j}$, number density $n_{j}$, and masses $m_{i}$ and $m_{j} . \Lambda$ is given by

$$
\Lambda=\frac{3}{2 Z_{i} Z_{j} e^{3}}\left(\frac{k^{3} T_{i}^{3}}{\pi n_{\mathrm{e}}}\right)^{\frac{1}{2}} .
$$

Solving the coupled differential equation given by Equation (3) for electrons, protons, $\mathrm{He}, \mathrm{O}, \mathrm{Si}$, and $\mathrm{Fe}$ (assuming solar abundances), we calculate temperature histories up to the measured $n_{\mathrm{e}} t$ for the measured proton temperature, for different electron temperatures at the shock front (Figure 5). This enables us to determine the electron temperature at the shock front (Table 1 and red data points in Figure 6).

Equation (1) shows that the proton temperature is related to the shock velocity. However, this is based on standard assumptions, assuming only a gaseous component. In contrast, supernova remnants have been suggested to efficiently accelerate particles which will alter the standard Hugoniot relations (Vink et al. 2010). The presence of X-ray synchrotron and TeV radiation indicates that $\mathrm{RCW} 86$ accelerates particles to high energies (Bamba et al. 2000; Borkowski et al. 2001; Vink et al. 2006; Aharonian et al. 2009), whereas the $\mathrm{H} \alpha$ line width in the NE shows that in this particular region cosmic-ray acceleration must be efficient, with a post-shock pressure contribution of more than 50\% (Helder et al. 2009).

When a shock accelerates cosmic rays, the cosmic rays create a cosmic-ray precursor ahead of the shock, pushing out and pre-heating the incoming medium. This effectively lowers the velocity of the material entering the main shock, therewith lowering the post-shock temperature (Drury et al. 2009; Hughes et al. 2000; Vink et al. 2010). Hence, $v_{\mathrm{s}}$ will not necessarily be the only variable to characterize $T_{\mathrm{p}}$. As we focus on $T_{\mathrm{e}}-T_{\mathrm{p}}$ equilibration, we plot $T_{\mathrm{e}}$ as function of the measured $T_{\mathrm{p}}$. This is more reliable as $v_{\mathrm{s}}$ is a quantity derived through Equation (1), which may not be valid in the presence of cosmic-ray acceleration (Helder et al. 2009).

Figure 6 shows that $T_{\mathrm{e}} \sim T_{\mathrm{p}}$ for the data point with the lowest proton temperatures. Surprisingly, the north $T_{\mathrm{e}}$ seems to be very high for the $T_{\mathrm{p}}$ based on the data from Ghavamian et al. (2007). However, Long \& Blair (1990) measured approximately the same position and found a broader broad component (overplotted in green in Figure 6). The rightmost data points in Figure 6 show $T_{\mathrm{e}}<T_{\mathrm{p}}$, consistent with previous results; however, we do not find the relation $T_{\mathrm{e}} / T_{\mathrm{p}} \propto 1 / v_{\mathrm{s}}^{2}$, which would result in a horizontal line, showed as a dashed line (Figure 6).

\section{CONCLUSIONS}

We investigated the proton-electron temperature equilibration behind several shock fronts of RCW 86. The low ionization parameter $n_{\mathrm{e}} t$ of this remnant ensures that the current $T_{\mathrm{e}}$ of the plasma is close that at the shock front. The electron temperatures were determined from X-ray spectra, obtained from $X M M-N e w t o n$ data and corrected for equilibration effects, resulting in Figure 6. We used both new and published data to determine proton temperatures. From this study, we can draw the following conclusions:

1. The FWHMs of the broad $\mathrm{H} \alpha$ line in the SE of RCW 86 are $1120 \pm 40$ and $920 \pm 50 \mathrm{~km} \mathrm{~s}^{-1}$ for the $S E_{\text {out }}$ and $S E_{\text {in }}$ spectra, respectively.

2. $T_{\mathrm{e}} / T_{\mathrm{p}} \sim 1$ for the slow shocks in the $\mathrm{E}$ and for the $\mathrm{N}$ parameters of Long \& Blair (1990). The N parameters of Ghavamian et al. (2007) and the SW form an exception to this.

3. $T_{\mathrm{e}} / T_{\mathrm{p}}<1$ for faster shocks. However, we do not find a constant electron temperature for faster shocks, as implied by the model of Ghavamian et al. (2007). Additionally, our results show $T_{\mathrm{e}} / T_{\mathrm{p}}>m_{\mathrm{e}} / m_{\mathrm{p}}$.

4. $T_{\mathrm{e}} / T_{\mathrm{p}}$ for the NE region, in which cosmic-ray acceleration appears to be efficient, is not well determined. However, we have found evidence for relatively high values of $T_{\mathrm{e}}$, suggesting that $T_{\mathrm{e}} / T_{\mathrm{p}}$ is close to one is this region. This may be attributed to efficient cosmic-ray acceleration, as this tends to lower the Mach number of the main shock.

We thank Andrei Bykov and John Raymond for useful discussions on shock physics and the interpretation of optical and X-ray spectra. E.A.H. and J.V. are supported by the Vidi grant of J.V. from the Netherlands Organization for Scientific Research (NWO).

\section{REFERENCES}

Aharonian, F., et al. 2009, ApJ, 692, 1500

Anders, E., \& Grevesse, N. 1989, Geochim. Cosmochim. Acta, 53, 197

Bamba, A., Koyama, K., \& Tomida, H. 2000, PASJ, 52, 1157

Borkowski, K. J., Rho, J., Reynolds, S. P., \& Dyer, K. K. 2001, ApJ, 550, 334

Bykov, A. M. 2004, Adv. Space Res., 33, 366

Bykov, A. M., \& Uvarov, Y. A. 1999, JETP, 88, 465

Cash, W. 1979, ApJ, 228, 939

Draine, B. T., \& McKee, C. F. 1993, ARA\&A, 31, 373

Drury, L. O., Aharonian, F. A., Malyshev, D., \& Gabici, S. 2009, A\&A, 496, 1 Ghavamian, P. 1999, PhD thesis, Rice Univ.

Ghavamian, P., Laming, J. M., \& Rakowski, C. E. 2007, ApJ, 654, L69

Ghavamian, P., Raymond, J., Smith, R. C., \& Hartigan, P. 2001, ApJ, 547, 995

Ghavamian, P., Winkler, P. F., Raymond, J. C., \& Long, K. S. 2002, ApJ, 572, 888

Helder, E. A., et al. 2009, Science, 325, 719

Heng, K. 2010, PASA, 27, 23 
Hughes, J. P., Rakowski, C. E., \& Decourchelle, A. 2000, ApJ, 543, L61

Kaastra, J. S., \& Jansen, F. A. 1993, A\&AS, 97, 873

Kaastra, J. S., Mewe, R., \& Nieuwenhuijzen, H. 1996, in UV and X-ray Spectroscopy of Astrophysical and Laboratory Plasmas, ed. K. Yamashita \& T. Watanabe (Tokyo: Universal Academy Press), 411

Kosenko, D., Helder, E. A., \& Vink, J. 2010, A\&A, 519, A11

Kosenko, D., Vink, J., Blinnikov, S., \& Rasmussen, A. 2008, A\&A, 490, 223

Long, K. S., \& Blair, W. P. 1990, ApJ, 358, L13

Rakowski, C. E., Ghavamian, P., \& Hughes, J. P. 2003, ApJ, 590, 846

Raymond, J. C., Vink, J., Helder, E. A., \& de Laat, A. 2011, ApJ, 731, L14

Rho, J., Dyer, K. K., Borkowski, K. J., \& Reynolds, S. P. 2002, ApJ, 581, 1116

Rybicki, G. B., \& Lightman, A. P. 1979, in Radiative Processes in Astrophysics, ed. G. B. Rybicki \& A. P. Lightman (New York: Wiley-Interscience)
Spitzer, L. (ed.) 1965, Physics of Fully Ionized Gases (Interscience Tracts on Physics and Astronomy; New York, NY: Interscience)

Strüder, L., et al. 2001, A\&A, 365, L18

Turner, M. J. L., et al. 2001, A\&A, 365, L27

van Adelsberg, M., Heng, K., McCray, R., \& Raymond, J. C. 2008, ApJ, 689, 1089

Vink, J., Bleeker, J., van der Heyden, K., Bykov, A., Bamba, A., \& Yamazaki, R. 2006, ApJ, 648, L33

Vink, J., Kaastra, J. S., \& Bleeker, J. A. M. 1997, A\&A, 328, 628

Vink, J., Yamazaki, R., Helder, E. A., \& Schure, K. M. 2010, ApJ, 722, 1727

Wheaton, W. A., Dunklee, A. L., Jacobsen, A. S., Ling, J. C., Mahoney, W. A., \& Radocinski, R. G. 1995, ApJ, 438, 322

Yamaguchi, H., et al. 2008, PASJ, 60, 123 